\title{
Opioid use during pregnancy: a population-based cohort study
}

\author{
Jamie Falk PharmD, Matthew Dahl BSc, Colette B. Raymond MSc PharmD, Dan Chateau PhD,
} Alan Katz MBChB MSc, Christine Leong PharmD, Shawn Bugden MSc PharmD

Abstract

Background: Opioid use has increased dramatically in North America. The safety of opioids in pregnancy is uncertain, but they are associated with several fetal abnormalities and contribute to rising rates of neonatal abstinence syndrome. We examined opioid use before and during pregnancy in a complete population-based cohort.

Methods: We examined opioid use in a cohort of all pregnant women in Manitoba, Canada, from 2001 to 2013 . Opioid use was defined by prescriptions for opioids, converted to oral morphine equivalents (MEQ), during the 3 months before pregnancy and for each trimester. Given that the exposure per person may vary (because not all women complete all time periods), we determined a weighted number of pregnancies in each period.

Results: During the study period, 174848 completed pregnancies were eligible for analysis (173 680 live births and 1168 stillbirths and intrauterine deaths), which represented a weighted value of 175174 pregnancies. Among these pregnancies, $6.7 \%$ of the women filled opioid prescriptions in the 3 months before pregnancy. Use declined to $4.2 \%$ during the first trimester and further declined to $3.0 \%$ and $2.9 \%$ in the second and third trimesters, respectively. Over the study period, there was a modest increase in opioid use overall (from $7.3 \%$ to $7.7 \%$ ). MEQ did not decline during pregnancy, and the mean MEQ increased significantly over the study period (from $284 \mathrm{mg}$ to $1218 \mathrm{mg}$ ). Prescriptions for codeine were filled by $96.9 \%$ of the users, accounting for $66.2 \%$ of $\mathrm{MEQ}$.

Interpretation: Although many of the women using opioids before pregnancy discontinued or reduced use of these drugs during pregnancy, the volume of opioids consumed by those who continued opioid use did not decline during pregnancy. The increasing dosage and increased use of higher-potency opioids by pregnant women highlights the need for continued evaluation of and education about the benefits and risks of this practice.

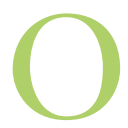

pioid use in North America has continued to increase dramatically over much of the past decade. ${ }^{1,2}$ Canada ranks with the United States as having the highest prescription opioid consumption in the world. ${ }^{3}$ Several US studies of health claims have found that opioid use in pregnancy has also increased over time. . $^{4-7}$ Recent studies have reported that $14 \%$ to $22 \%$ of women filled at least 1 prescription for an opioid during pregnancy, with exposures as high as $42 \%$ in some US states. ${ }^{5,6,8}$ Although the effects of opioid use on the developing fetus are poorly understood and findings are varied, associations with complications, including neural tube defects and cardiac septal defects, have been found. ${ }^{9-11}$ Neonatal abstinence syndrome, a drug-withdrawal syndrome that most commonly occurs after in utero exposure to opioids, has become one of the fastest growing reasons for neonatal hospital admissions. ${ }^{11,12}$ Recent studies of opioid exposure in pregnancy have reported incidences of 4.3 to 5.9 cases of neonatal abstinence syndrome per 1000 births. ${ }^{13,14}$
Considering the overall population use of opioids in North America and the potential harms of opioid exposure during pregnancy to the developing fetus and the neonate, it is important to study prescribing patterns in order to understand the potential implications from a public health perspective. Two large retrospective analyses of outcomes related to antenatal and postpartum opioid use have been performed in Ontario, Canada, in addition to small cohort studies evaluating the incidence of narcotic abuse during pregnancy in communities in northwestern Ontario. ${ }^{13,15-17}$ The objectives of this study were to determine, for the period 2001 to 2013,

\section{Competing interests: None declared.}

This article has been peer reviewed.

Correspondence to: Shawn Bugden, Shawn.Bugden@umanitoba.ca

CMAJ Open 2017. DOI:10.9778/cmajo.20160102 
the frequency of use and volume of consumption of opioids among pregnant women in Manitoba, Canada, before and during pregnancy and to determine the rate of continued use of opioid agents through each trimester.

\section{Methods}

\section{Setting and data sources}

We performed an observational study of pregnancy and opioid use using administrative health care claims data from the Population Health Research Data Repository, housed at the Manitoba Centre for Health Policy. We used the Medical Services database, which captures all fee-for-service medical claims, to determine the date of first pregnancy-related physician visit. We also used the Hospital Discharge Abstract database, which contains summary data for each hospital stay; the Manitoba Health Registry, which contains basic demographic information on persons registered with Manitoba Health; and the Drug Program Information Network, for prescription drug claims. We used neighbourhood income quintiles from public-use census files to determine socioeconomic status. These data sets are linked at the person level with an encrypted identifier and have been used extensively in health services research. ${ }^{18-23}$

\section{Design}

We created a cohort of all pregnant women in Manitoba with resulting live birth, stillbirth or intrauterine death who were registered with Manitoba Health during the pregnancy, in the year before the pregnancy and 3 months after the delivery. We identified pregnancies by codes from the International Classification of Diseases, 9th revision (V27.0-V27.9, 656.4) and corresponding codes from the International Statistical Classification of Diseases and Related Health Problems, 10th revision (Z37.0-Z37.9, O36.4). ${ }^{24}$ We included women with more than 1 pregnancy during the study period as multiple pregnancy observations, and excluded molar and ectopic pregnancies and pregnancies ending in abortion. Because of uncertainty about delivery date, we excluded women with length of stay in hospital more than 7 days and no newborn data that would allow assignment of a birth date..$^{25}$ Gestational age is available through hospital discharge data (maternal and newborn records) for $98.6 \%$ of live births, stillbirths and intrauterine deaths. We determined date of conception by subtracting gestational age at delivery from the maternal hospital admission date. ${ }^{25}$

We considered the pre-pregnancy period ( 3 mo before conception) and each trimester of pregnancy. Given that the exposure per person may vary (because not all women complete all time periods), we determined a weighted number of pregnancies in each period. We summed the actual number of days each woman contributed during a given period for all pregnancies, and then divided the total number of days that all pregnancies contributed to a period by the possible number of days in that period (e.g., $91 \mathrm{~d}$ for each trimester). Inclusion in the opioid user cohort was defined by filling of prescriptions for opioids at any time after date of conception. We analyzed this cohort for patterns of use over time starting 3 months before pregnancy and through each trimester. To evaluate opioid use by women who would be certain they were pregnant, we performed a comparative analysis in which inclusion in the user cohort was defined by filling of prescriptions for opioids after the first pregnancy-related visit. In-hospital medication use was not available and was therefore not included in the study.

For each opioid prescription, we calculated the total amount dispensed by multiplying the total number of tablets (or total volume of liquid) by the strength of the pills (or concentration of the liquid). We then converted the calculated total opioid (in milligrams) to oral morphine equivalents (MEQ) using the morphine equivalence ratios of the Canadian Guideline for Safe and Effective Use of Opioids for Chronic NonCancer Pain ${ }^{26}$ with supplementary references used for opioids not listed in the Canadian guideline. ${ }^{27,28}$ We excluded methadone from the analysis because most methadone use was in the form of compounded products that did not allow reliable determination of the actual quantity of medication.

\section{Statistical analysis}

We used $\chi^{2}$ and $t$ tests to compare opioid use at the beginning and end of the study period. To evaluate trends in rates of use over the study period, we ran Poisson regression models with $\log$ of the population as the offset. To evaluate opioid use across pregnancy trimesters, we ran logistic regression generalized estimating equation models; these models accounted for the correlated observations (i.e., repeated measures) of individuals across the pregnancy. All analyses were performed with SAS version 9.3 (SAS Institute Inc.).

\section{Ethics approval}

Approval for this study was obtained from the University of Manitoba Health Research Ethics Board (HREB HS16618 [H2013:332]).

\section{Results}

During the study period, there were a total of 245704 pregnancies. There were 49914 exclusions for molar and ectopic pregnancies and abortions, and 5633 exclusions related primarily to multiple pregnancy outcomes per event date and nonavailability of gestational age. Women not having continuous registration in Manitoba for health coverage accounted for an additional 15309 exclusions. After these exclusions, there were 174848 completed pregnancies eligible for analysis (173 680 live births and 1168 stillbirths and intrauterine deaths), which represented a weighted value of 175174 pregnancies.

Over the study period, an opioid was dispensed for 13408 women $(7.7 \%)$ during their pregnancy. The median age was 28 years for the overall cohort and 27 years for the cohort with exposure to opioids. The incident pregnancy was the first pregnancy for $29.3 \%$ of women in the overall cohort and for $18.5 \%$ of those with exposure to opioids. The lowest income quintile accounted for $26.3 \%$ of the overall cohort, compared with $40.0 \%$ of those with exposure to opioids (Table 1). 


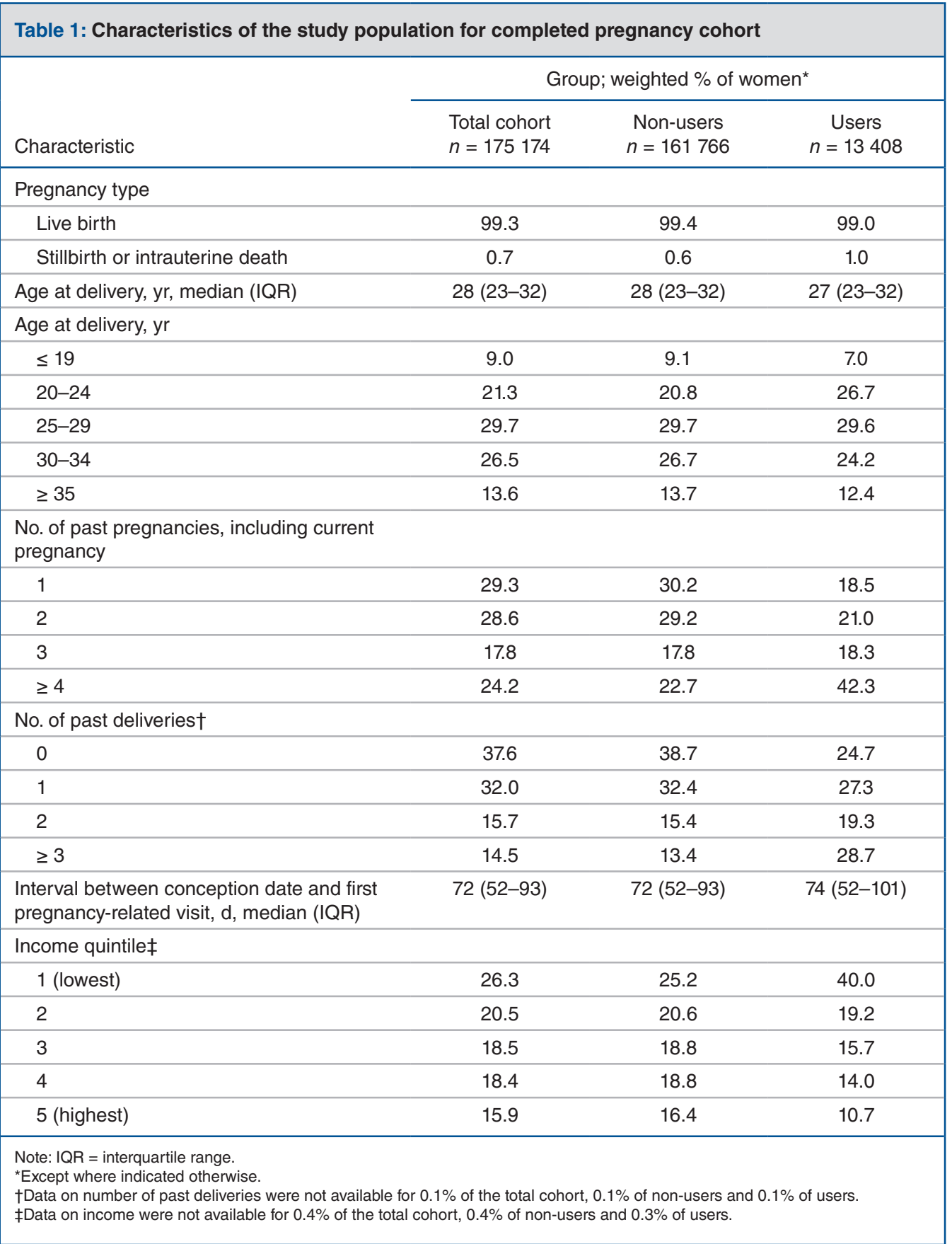

Over the study period, there was a modest increase in the proportion of women who filled prescriptions for opioids at any time during pregnancy, from $7.3 \%$ in 2001 to $7.7 \%$ in $2013(p=0.03)$ (Figure 1). In the 3 months before pregnancy, an opioid was dispensed for 11692 women (6.7\%). Opioid use fell to $4.2 \%$ during the first trimester of pregnancy and further declined to $3.0 \%$ in the second and $2.9 \%$ in the third trimester $(p<0.001$ for each trimester compared with the 3 mo before pregnancy and for second and third trimesters compared with the first trimester). A similar pattern of usage decline from the pre-pregnancy period through the trimesters was observed from the start to the end of the study period (Figure 1).
There was a significant increase in consumption of prescription opioids across the study period. For women who filled prescriptions for opioids at any time during pregnancy, the mean MEQ per pregnancy increased more than 4-fold over the study period, from $284 \mathrm{mg}$ in 2001 to $1218 \mathrm{mg}$ in $2013(p<0.001)$ (Figure 2). The mean MEQ over the 3 months before pregnancy was $421 \mathrm{mg}$, similar to the $493 \mathrm{mg}, 545 \mathrm{mg}$ and $511 \mathrm{mg}$ used in the first, second and third trimesters of pregnancy, respectively $(p>0.9$ for all comparisons). A similar pattern from the pre-pregnancy period through the trimesters was observed from the start to the end of the study period (Figure 2). 


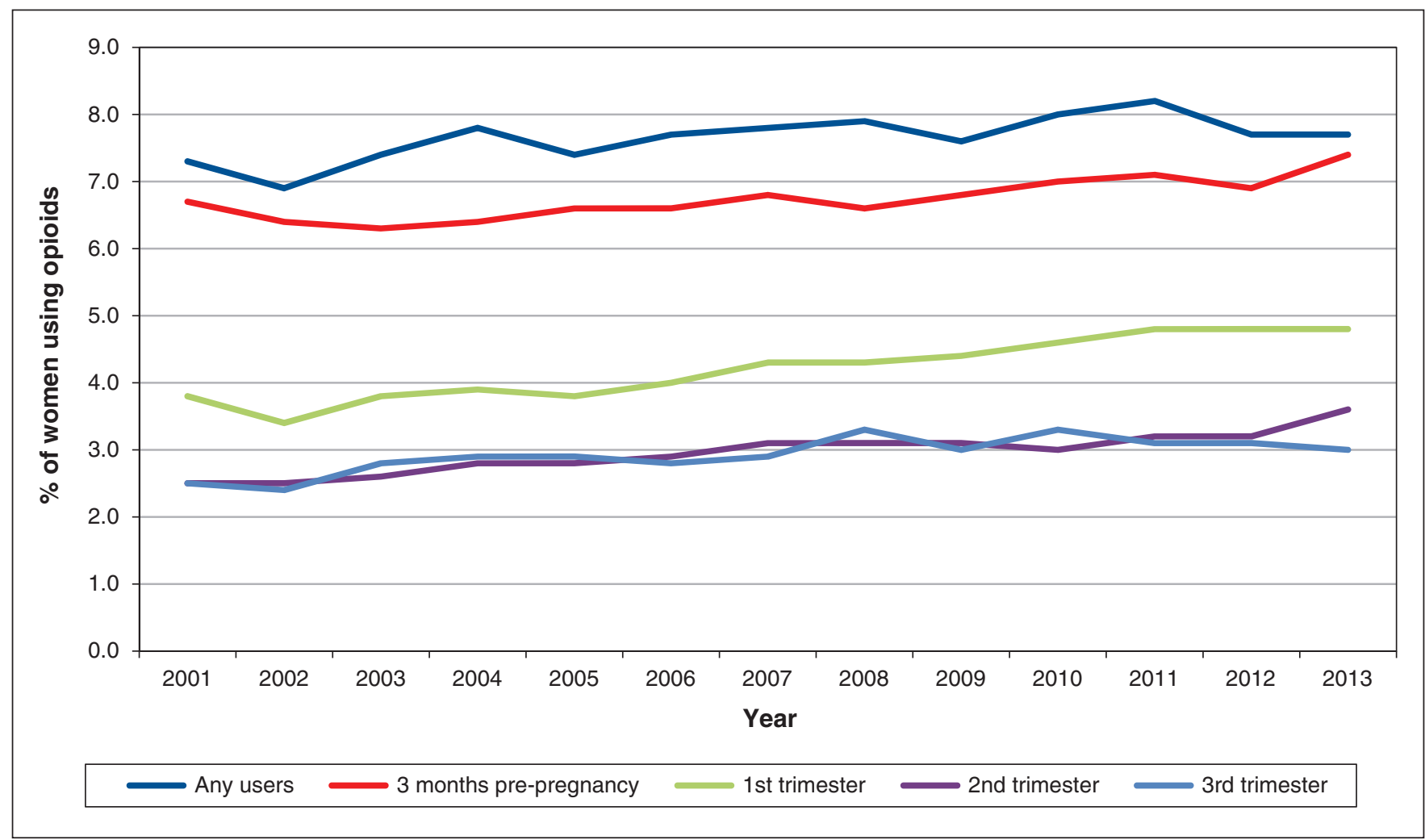

Figure 1: Opioid use for overall pregnancy cohort over study period.

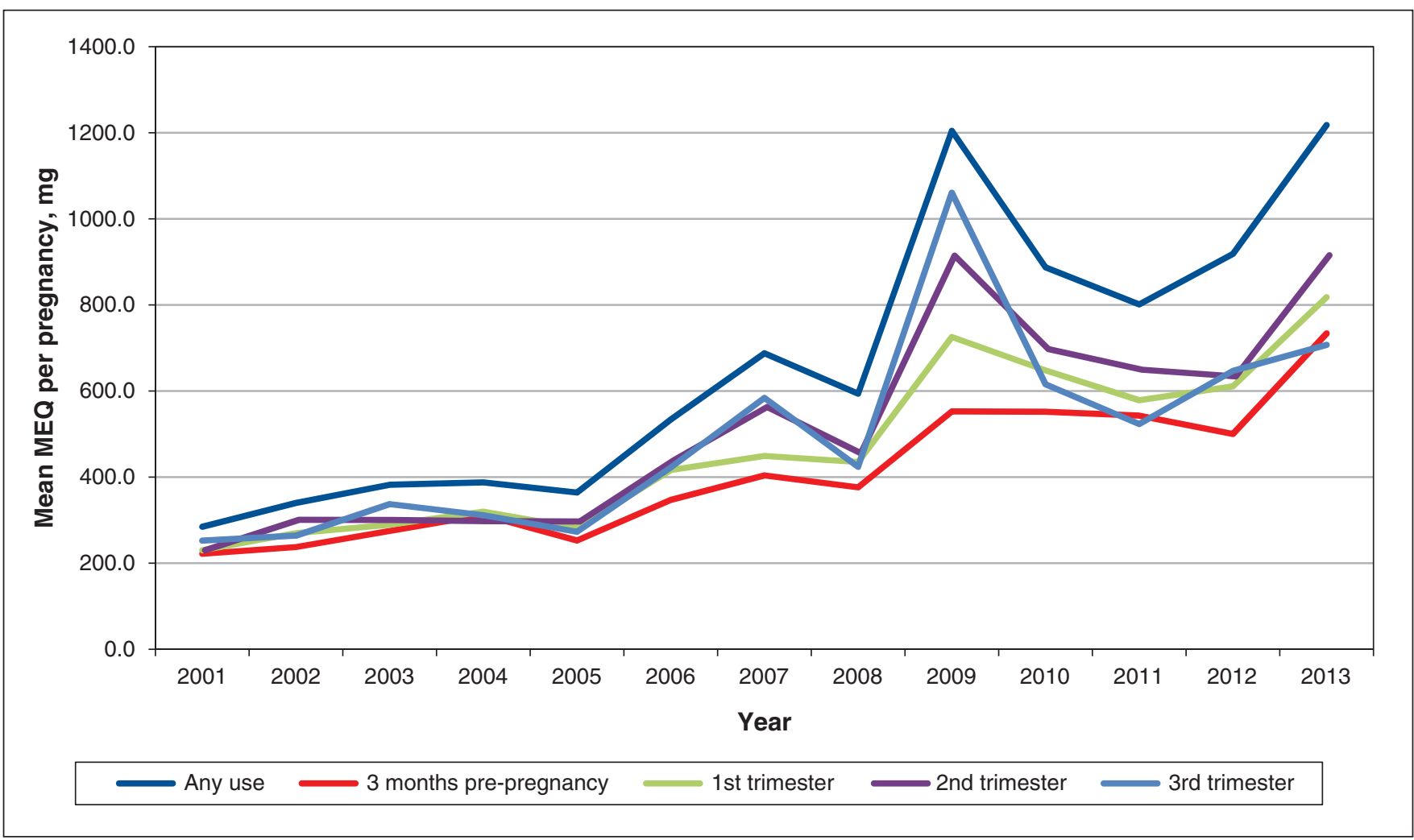

Figure 2: Mean opioid use, as morphine equivalents (MEQ), for opioid users over the study period. Mean MEQ = total MEQ divided by total weighted number of pregnancies among opioid users. 
In the comparative analysis with cohort inclusion defined as filling of any opioid prescription after the first pregnancyrelated visit, overall use of opioids was lower than in the primary analysis (4.9\%), but a similar pattern of use and consumption was seen over the study period. Use increased modestly from $4.3 \%$ in 2001 to $4.8 \%$ in 2013 ( $p=0.01$ ), while mean MEQ increased from $281 \mathrm{mg}$ in 2001 to $1046 \mathrm{mg}$ in $2013(p<0.001)$.

Of women in the lowest and highest income quintiles, $11.7 \%$ and $5.1 \%$ were opioid users, respectively $(p<0.001)$. The mean MEQ during pregnancy did not differ significantly between these 2 quintile groups (773 mg v. $653 \mathrm{mg}, p=0.5$ ) (Table 2).

Codeine accounted for most of the opioid prescriptions, having been dispensed to $96.9 \%$ of all exposed women and accounting for $66.2 \%$ of MEQ. The proportion of the MEQ accounted for by codeine declined over the study period, from
$87.8 \%$ in 2001 to $56.7 \%$ in 2013 . There was a corresponding increase in the contribution of higher-potency opioids to the MEQ over this time period (Table 3).

\section{Interpretation}

Among the more than 175000 pregnancies in the population of Manitoba, Canada (2001-2013), an opioid was dispensed for $7.7 \%$ of women during the pregnancy. Over the study period, the proportion of those who filled prescriptions for opioids in pregnancy increased modestly; however, the amount of prescription opioid used during pregnancy increased dramatically. The proportion of women using prescription opioids declined from the pre-pregnancy period to the first trimester and further declined into the second trimester; however, the volume of opioids used by those who continued opioid use did not decline.

\begin{tabular}{|c|c|c|c|c|}
\hline \multirow[b]{2}{*}{$\begin{array}{l}\text { Income } \\
\text { quintile }\end{array}$} & \multicolumn{2}{|c|}{ Opioid use, \% } & \multicolumn{2}{|r|}{ Average $\mathrm{MEQ}, \mathrm{mg}$} \\
\hline & $\begin{array}{l}\text { Any time during } \\
\text { pregnancy }\end{array}$ & $\begin{array}{l}\text { After first pregnancy- } \\
\text { related visit }\end{array}$ & Overall & $\begin{array}{c}\text { Those using opioids after first } \\
\text { pregnancy-related visit }\end{array}$ \\
\hline 1 (lowest) & $11.7^{\star}$ & $6.9^{*}$ & 773† & $689 \ddagger$ \\
\hline 2 & 7.2 & 4.6 & 720 & 615 \\
\hline 3 & 6.5 & 4.3 & 543 & 497 \\
\hline 4 & 5.8 & 3.9 & 562 & 486 \\
\hline 5 (highest) & $5.1^{*}$ & $3.7^{*}$ & 653† & $668 \ddagger$ \\
\hline \multicolumn{5}{|c|}{$\begin{array}{l}\text { Note: } \mathrm{MEQ}=\text { morphine equivalents. } \\
{ }^{*} p<0.001 \text { for comparison of quintiles } 1 \text { and } 5 . \\
t p=0.5 \text { for comparison of quintiles } 1 \text { and } 5 . \\
\neq p=0.9 \text { for comparison of quintiles } 1 \text { and } 5 .\end{array}$} \\
\hline
\end{tabular}

\begin{tabular}{|c|c|c|c|c|}
\hline \multirow[b]{2}{*}{ Opioid } & \multicolumn{2}{|c|}{2001} & \multicolumn{2}{|c|}{2013} \\
\hline & $\begin{array}{c}\text { Use, } \\
\text { no. (\%) of women* }\end{array}$ & $\begin{array}{l}\text { MEQ, } \\
\% \text { of total }\end{array}$ & $\begin{array}{c}\text { Use, } \\
\text { no. (\%) of women* }\end{array}$ & $\begin{array}{c}\text { MEQ, } \\
\% \text { of total }\end{array}$ \\
\hline Any opioid $†$ & $n=937$ & & $n=1132$ & \\
\hline Codeine & $922(98.4)$ & 87.8 & $1069(94.4)$ & 56.7 \\
\hline Fentanyl & $0(0)$ & - & $S(<1)$ & $<1.0$ \\
\hline Hydrocodone & $S(<1.0)$ & $<1.0$ & $0(0)$ & - \\
\hline Hydromorphone & $S(<1.0)$ & $<1.0$ & $11(1.0)$ & 5.3 \\
\hline Meperidine & 15 (1.6) & 4.3 & $S(<1.0)$ & $<1.0$ \\
\hline Morphine & $S(<1.0)$ & 6.6 & $26(2.3)$ & 18.8 \\
\hline Oxycodone & $S(<1.0)$ & $<1.0$ & 49 (4.3) & 18.2 \\
\hline Tramadol & $0(0)$ & - & $29(2.6)$ & $<1.0$ \\
\hline
\end{tabular}


Codeine was used by most of these women, and use of this drug showed a small decline over the study period. The proportion of total MEQ accounted for by codeine decreased significantly over the study period, with a corresponding increase in more powerful opioids. Although we saw a modest increase in the proportion of opioid users over time, more striking was the finding, based on an analysis of MEQ, of a dramatic increase in the overall volume of opioids dispensed; this result suggests that women who used opioids during pregnancy were using more or were switching away from less potent options, such as codeine, to more potent forms, such as morphine and oxycodone, which would make a larger contribution to the MEQ. To our knowledge, no population-based Canadian studies have evaluated the volume of opioids used in pregnancy on the basis of MEQ consumed. Considering that opioids have been associated with neural tube defects, cardiac septal defects and the rise in neonatal hospital admissions associated with neonatal abstinence syndrome, our findings raise some concern. ${ }^{9-14}$

A previous analysis of the overall Canadian population, for the period 2005-2010, showed a similar increase in opioid use of $13.1 \% .^{29}$ US database studies have also shown increases in opioid use during pregnancy (from the mid-1990s to 2009), but the magnitude of change was generally larger than what we found, with increases in exposure ranging from 0.23 -fold to 4.73 -fold. ${ }^{4-7}$ The most up-to-date exposure rates in the US were varied but generally much higher than in Manitoba, ranging from $14 \%$ to $42 \%$, with pharmacy dispensing claims data being used to determine opioid exposure. ${ }^{5,6,8}$

There are several possible reasons for the differences in opioid use between US pharmacy claims studies and our findings. These differences may indicate substantially greater use of opioids by pregnant women in the US. Manitoba has a fully linked drug information network that allows all pharmacists to review any prescription filled in the past and requires a special physician-specific, fraud-resistant prescription for singleentity opioid prescriptions. The variation between studies may also be related to characteristics of the populations studied. Rates of prescription opioid use have been reported to be higher among those enrolled in Medicaid than among commercially insured patients. ${ }^{30}$ However, Bateman and colleagues, ${ }^{8}$ despite having one of the lower estimates among the current US studies, reported a considerably higher rate of prenatal opioid use (14\% in a commercially insured US population) than was found in our study. In Manitoba, those with lower economic status have full medication coverage, with minimal or no deductible. ${ }^{31}$ Thus, our study population, which encompassed all residents in the province, incorporated aspects of both the commercially insured and publicly insured US populations. Our analysis of income quintiles showed that those in the lowest income group had a significantly higher rate of use $(11.7 \%)$ than those in the highest income group, although still lower than rates found in US studies.

The findings of Handal and colleagues, ${ }^{32}$ who reported the prevalence of opioid use among pregnant women in Norway between 2004 and 2008 as only 3\%, were more similar to our results. Similar to our study, use of codeine accounted for most exposures, at $91 \%$. In addition, codeine use declined from $2.4 \%$ to $0.93 \%$ from 3 months before pregnancy to the second trimester, a relative reduction of about $60 \%$, which was very similar to that found in our overall pregnancy cohort (from $6.7 \%$ to $3.0 \%$ ). Instead of using MEQ as a marker of volume of use, Handal and colleagues ${ }^{32}$ assessed divided daily doses (DDD), finding a small reduction from 6.7 DDD in the 3 months before pregnancy to $5 \mathrm{DDD}$ in the second and third trimesters. This result contrasts with our study, using MEQ as the marker, which showed no reduction in volume of use.

\section{Limitations and strengths}

Our study had certain limitations. First, determining medication use from pharmacy dispensing claims does not allow assessment of whether the women actually took the medication. In addition, use of pharmacy claims data does not allow capture of use of over-the-counter opioid analgesics that contain low doses of codeine, illicit opioid use or inpatient opioid use; as a result, the true extent of opioid use would be underestimated.

Use of the Manitoba Centre for Health Policy database offered several advantages. First, it allowed a Canadian perspective on frequency and utilization patterns of opioid use by pregnant women. Second, data from the Drug Program Information Network accounts for all women, regardless of socioeconomic status, and linkage to socioeconomic status variables allowed for comparisons among socioeconomic strata of the population.

\section{Conclusion}

In this study population, many women who used opioids before pregnancy discontinued their use during pregnancy. Although the percentage of opioid users in pregnancy was low and declined across trimesters relative to pre-pregnancy values, the volume of opioids used by those who continued to use these drugs remained constant, relative to the pre-pregnancy period, and increased significantly over time. Given this increasing volume of opioid use over time, these findings indicate a need for continued evaluation of the safety of this group of medications during pregnancy and necessitate the provision of better information regarding the currently understood benefits and risks of pregnancy-related opioid use to guide clinical care.

\section{References}

1. Gomes T, Mamdani M, Paterson JM, et al. Trends in high-dose opioid prescribing in Canada. Can Fam Physician 2014;60:826-32.

2. Levy B, Paulozzi L, Mack KA, et al. Trends in opioid analgesic-prescribing rates by specialty, U.S., 2007-2012. Am 7 Prev Med 2015;49:409-13.

3. Opioid consumption motion chart. Madison (WI): University of Wisconsin System, Board of Regents; 2013. Available: https://ppsg.medicine.wisc.edu/ chart (accessed 2016 May 20).

4. Creanga AA, Sabel JC, Ko JY, et al. Maternal drug use and its effect on neonates: a population-based study in Washington State. Obstet Gynecol 2012; 119:924-33.

5. Epstein RA, Bobo WV, Martin PR, et al. Increasing pregnancy-related use of prescribed opioid analgesics. Ann Epidemiol 2013;23:498-503.

6. Desai RJ, Hernandez-Diaz S, Bateman BT, et al. Increase in prescription opioid use during pregnancy among Medicaid-enrolled women. Obstet Gynecol 2014;123:997-1002.

7. Patrick SW, Schumacher RE, Benneyworth BD, et al. Neonatal abstinence syndrome and associated health care expenditures: United States, 2000-2009. fAMA 2012;307:1934-40. 
8. Bateman BT, Hernandez-Diaz S, Rathmell JP, et al. Patterns of opioid utilization in pregnancy in a large cohort of commercial insurance beneficiaries in the United States. Anesthesiology 2014;120:1216-24.

9. Broussard CS, Rasmussen SA, Reefhuis J, et al.; National Birth Defects Prevention Study. Maternal treatment with opioid analgesics and risk for birth defects. Am $\mathcal{f}$ Obstet Gynecol 2011;204:314.e1-11.

10. Yazdy MM, Mitchell AA, Tinker SC, et al. Periconceptional use of opioids and the risk of neural tube defects. Obstet Gynecol 2013;122:838-44.

11. Anand KJS, Campbell-Yeo M. Consequences of prenatal opioid use for newborns. Acta Paediatr 2015;104:1066-9.

12. Tolia VN, Patrick SW, Bennett MM, et al. Increasing incidence of the neonatal abstinence syndrome in U.S. neonatal ICUs. N Engl 7 Med 2015;372:2118-26.

13. Turner SD, Gomes T, Camacho X, et al. Neonatal opioid withdrawal and antenatal opioid prescribing. CMA7 Open 2015;3:E55-61.

14. Desai RJ, Huybrechts KF, Hernandez-Diaz S, et al. Exposure to prescription opioid analgesics in utero and risk of neonatal abstinence syndrome: population based cohort study. BM7 2015;350:h2102.

15. Juurlink DN, Gomes T, Guttmann A, et al. Postpartum maternal codeine therapy and the risk of adverse neonatal outcomes: a retrospective cohort study. Clin Toxicol (Phila) 2012;50:390-5.

16. Kelly L, Dooley J, Cromarty H, et al. Narcotic-exposed neonates in a First Nations population in northwestern Ontario: incidence and implications. Can Fam Physician 2011;57:e441-7.

17. Kelly L, Guilfoyle J, Dooley J, et al. Incidence of narcotic abuse during pregnancy in northwestern Ontario: three-year prospective cohort study. Can Fam Physician 2014;60:e493-8.

18. Roos NP, Shapiro E. Revisiting the Manitoba Centre for Health Policy and Evaluation and its population-based health information system. Med Care 1999;37(6 Suppl):JS10-4.

19. Roos LL, Nicol JP. A research registry: uses, development, and accuracy. 7 Clin Epidemiol 1999:52:39-47.

20. Roos LL, Brownell M, Lix L, et al. From health research to social research: privacy, methods, approaches. Soc Sci Med 2008;66:117-29.

21. Roos LL Jr, Nicol JP, Cageorge SM. Using administrative data for longitudinal research: comparisons with primary data collection. 7 Chronic Dis 1987;40:41-9.

22. Roos LL, Gupta S, Soodeen RA, et al. Data quality in an information-rich environment: Canada as an example. Can 7 Aging 2005;24(Suppl 1):153-70.

23. Robinson JR, Young TK, Roos LL, et al. Estimating the burden of disease. Comparing administrative data and self-reports. Med Care 1997;35:932-47.

24. Heaman M, Kingston D, Helewa ME, et al. Perinatal services and outcomes in Manitoba. Winnipeg: Manitoba Centre for Health Policy; 2012.

25. Daw JR, Mintzes B, Law MR, et al. Prescription drug us in pregnancy: a retrospective population based study in British Columbia Canada (2001-2006). Clin Ther 2012;34:239-49.e2.

26. National Opioid Use Guideline Group. Canadian guideline for safe and effective use of opioids for chronic non-cancer pain. Hamilton (ON): McMaster University; 2010. Available: http://nationalpaincentre.mcmaster.ca/opioid/ (accessed 2016 June 15).

27. Gammaitoni AR, Fine P, Alvarez N, et al. Clinical application of opioid equianalgesic data. Clin 7 Pain 2003;19:286-97.
28. Stanford School of Medicine, Palliative Care. Opioid conversion: equivalency table. Stanford (CA): Stanford School of Medicine; 2017. Available: https://palliative. stanford.edu/opioid-conversion/equivalency-table/ (accessed 2017 Jan. 12).

29. Fischer B, Jones W, Krahn M, et al. Differences and over-time changes in levels of prescription opioid analgesic dispensing from retail pharmacies in Canada, 2005-2010. Pharmacoepidemiol Drug Saf 2011;20:1269-77.

30. Sullivan MD, Edlund MJ, Fan MY, et al. Trends in use of opioids for noncancer pain conditions 2000-2005 in commercial and Medicaid insurance plans: the TROUP study. Pain 2008;138:440-9.

31. About the Manitoba Pharmacare Program. Winnipeg: Manitoba Health, Seniors and Active Living. Available: www.gov.mb.ca/health/pharmacare/ index.html (accessed 2016 May 26).

32. Handal M, Engeland A, Rønning M, et al. Use of prescribed opioid analgesics and co-medication with benzodiazepines in women before, during, and after pregnancy: a population-based cohort study. Eur 7 Clin Pharmacol 2011;67:953-60

Affiliations: College of Pharmacy, Rady Faculty of Health Sciences (Falk, Leong, Bugden), and Manitoba Centre for Health Policy, Department of Community Health Sciences (Dahl, Raymond, Chateau, Katz), University of Manitoba, Winnipeg, Man.

Contributors: All of the authors were involved in study conception and design and acquisition of the data. Jamie Falk, Matthew Dahl and Shawn Bugden performed the initial analysis and interpretation of the data. All of the authors provided comment on refinement of the analysis and data interpretation. Jamie Falk and Shawn Bugden drafted the manuscript. All of the authors revised and critically evaluated the manuscript, approved the final version for publication and agreed to act as guarantors of the work.

Funding: This study was funded through the University of Manitoba.

Acknowledgements: The authors acknowledge the Manitoba Centre for Health Policy for use of data contained in the Population Health Research Data Repository, under project HIPC 2013/2014-35. The results and conclusions are those of the authors, and no official endorsement by the Manitoba Centre for Health Policy, Manitoba Health, Seniors and Active Living, or other data providers is intended or should be inferred. Data used in this study are from the Population Health Research Data Repository housed at the Manitoba Centre for Health Policy, University of Manitoba, and were derived from data provided by Manitoba Health, Seniors and Active Living.

Supplemental information: For reviewer comments and the original submission of this manuscript, please see www.cmajopen.ca/content/5/2/ E517/suppl/DC1 